\title{
Les hommes-relais de la bactériologie en territoire québécois et l'introduction de nouvelles pratiques diagnostiques et thérapeutiques (1890-1920)
}

\section{Denis Goulet et Othmar Keel}

Volume 46, numéro 3, hiver 1993

URI : https://id.erudit.org/iderudit/305108ar

DOI : https://doi.org/10.7202/305108ar

Aller au sommaire du numéro

Éditeur(s)

Institut d'histoire de l'Amérique française

ISSN

0035-2357 (imprimé)

1492-1383 (numérique)

Découvrir la revue

Citer cet article

Goulet, D. \& Keel, O. (1993). Les hommes-relais de la bactériologie en territoire québécois et l'introduction de nouvelles pratiques diagnostiques et thérapeutiques (1890-1920). Revue d'histoire de l'Amérique française, 46(3), 417-442. https://doi.org/10.7202/305108ar

\section{Résumé de l'article}

Cet article rend compte de l'introduction de certaines pratiques bactériologiques au sein du corps médical au Québec entre les années 1890 et 1920. Sont présentés ici les premiers hommes-relais de la bactériologie initiés pour la plupart en territoire européen à la théorie et aux pratiques bactériologiques, ainsi que les premiers cours de bactériologie dispensés au sein des facultés médicales. Est aussi prise en considération, l'émergence des méthodes bactériologiques au sein de la pratique clinique et de la pratique hospitalière. Mais alors que les activités de recherche en ce domaine demeureront peu développées, l'introduction de ces nouvelles méthodologies diagnostiques par le biais de l'enseignement et de la clinique hospitalière s'est effectuée sans retard véritable par rapport à la communauté médicale internationale. Elles ont même largement contribué à développer une médecine de laboratoire.
Tous droits réservés @ Institut d'histoire de l'Amérique française, 1993
Ce document est protégé par la loi sur le droit d'auteur. L'utilisation des services d’Érudit (y compris la reproduction) est assujettie à sa politique d'utilisation que vous pouvez consulter en ligne. 


\title{
LES HOMMES-RELAIS DE LA BACTÉRIOLOGIE EN TERRITOIRE QUÉBÉCOIS ET L'INTRODUCTION DE NOUVELLES PRATIQUES DIAGNOSTIQUES ET THÉRAPEUTIQUES $(\mathbf{1 8 9 0 - 1 9 2 0})^{1}$
}

\author{
DENIS GOULET \\ OTHMAR KEEL \\ Département d'histoire \\ Université de Montréal
}

\section{RÉSUMÉ}

Cet article rend compte de l'introduction de certaines pratiques bactériologiques au sein du corps médical au Québec entre les années 1890 et 1920 . Sont présentés ici les premiers hommes-relais de la bactériologie initiés pour la plupart en territoire européen à la théorie et aux pratiques bactériologiques, ainsi que les premiers cours de bactériologie dispensés au sein des facultés médicales. Est aussi prise en considération, l'émergence des méthodes bactériologiques au sein de la pratique clinique et de la pratique hospitalière. Mais alors que les activités de recherche en ce domaine demeureront peu développées, l'introduction de ces nouvelles méthodologies diagnostiques par le biais de l'enseignement et de la clinique hospitalière s'est effectuée sans retard véritable par rapport à la communauté médicale internationale. Elles ont même largement contribué à développer une médecine de laboratoire.

\begin{abstract}
This paper presents an account of the adoption by the medical profession in Quebec, during the years 1890 to 1920, of certain germ-fighting practices. It introduces to the first shifts of men trained, for most of them on the Continent, in the theory and practice of bacteriology. It describes also the first bacteriology courses in the faculties of medicine as well as the emergence of bacteriological methodologies in the hospital and clinical practice. The new diagnostic methods introduced in the medical courses or in the hospital clinical setting indeed made their appearance without any significant delay with regard to what happened in the international medical community. Moreover, even if the diagnostic activities then prevailed at the expense of research, they nevertheless contributed widely to the development of laboratory medicine.
\end{abstract}

1. Cette recherche a bénéficié d'une aide financière du Conseil de recherches en sciences humaines du Canada et du Fonds FCAR du Québec. 


\section{INTRODUCTION}

Tout au long du XIX ${ }^{e}$ siècle, les maladies infectieuses constituent au Québec la principale cause de mortalité. Les moyens mis en œuvre pour les contrôler ou les éradiquer se sont modifiés en fonction de facteurs externes (économique, social, politique), et de facteurs internes (théories étiologiques, procédés diagnostiques et thérapeutiques). Les modifications des conceptions étiologiques des maladies infectieuses produites par la bactériologie pénètrent en territoire québécois durant les deux dernières décennies du $\mathrm{XIX}^{\mathrm{e}}$ siècle. Nous avons montré ailleurs ${ }^{2}$ que les premiers échos de la théorie des germes durant la décennie 1870 se rapportent surtout aux pratiques chirurgicales. Les thèses bactériologiques demeurent encore marginales au sein d'une profession médicale qui se rallie plutôt à des positions éclectiques où les miasmes, germes, animalcules, poisons morbides et perturbations cosmiques sont envisagés comme des causes possibles des maladies infectieuses. De telles figures du discours médical, loin d'être de pures spéculations, constituaient un cadre épistémologique cohérent qui permettait de rendre compte en partie des phénomènes épidémiques.

C'est durant la décennie 1880 que les thèses étiologiques défendues au Québec suivent les développements du savoir médical international en matière de maladies contagieuses. Les «microbes» ${ }^{3}$ observés dans les laboratoires de Pasteur ou de Koch se substituent peu à peu aux miasmes et aux contages. Le phénomène de la contagion, qui avait donné lieu à de nombreuses recherches et à de nombreuses hypothèses tout au long du $\mathrm{XIX}^{\mathrm{e}}$ siècle, se trouvait en partie élucidé. Elle était entendue comme une intrusion d'êtres vivants microscopiques susceptibles de se multiplier chez l'hôte et de se propager à l'aide de vecteurs aériens, hydriques, humains, animaux, etc. Il était désormais possible de reconnaître en laboratoire et parfois dans le cabinet du médecin, certains micro-organismes responsables de maladies spécifiques. Le champ de la prophylaxie subit des modifications alors même que certaines mesures préventives élaborées avant les démonstrations bactériologiques reçoivent une légitimité accrue. L'amélioration du vaccin antivariolique, la mise au point du sérum antidiphtérique, les tests de dépistage des maladies vénériennes, la connaissance du rôle joué par les staphylocoques et les streptocoques

2. D. Goulet et $\mathrm{O}$. Keel, «Généalogie des représentations et attitudes face aux épidémies au Québec depuis le XIX ${ }^{e}$ siècle», Anthropologie et Sociétés, (L'univers du SIDA) (1991): 205228. Voir aussi D. Goulet et $O$. Keel, «L'impact de la bactériologie sur la pratique chirurgicale au Québec. 1. L'introduction des pratiques antiseptiques (1868-1890)», Bulletin canadien d'histoire de la médecine/Canadian Bulletin of the History of Medicine, soumis pour publication. en 1878 .

3. Rappelons que le mot microbe est proposé par C. Sédillot à l'Académie des sciences 
dans les infections opératoires ont certes contribué à établir les paramètres d'une nouvelle médecine préventive.

Alors que la théorie des germes est de plus en plus reconnue par la majorité des élites de la profession médicale - hygiénistes, médecins, chirurgiens - durant les décennies 1880 et 1890 , comme fondement de la prévention du processus infectieux, les procédés diagnostiques et thérapeutiques de la bactériologie ne se sont véritablement implantés au Québec qu'au tournant du $\mathrm{XX}^{\mathrm{e}}$ siècle ${ }^{4}$. Antérieurement, les revues médicales publiaient certes de nombreux extraits des travaux européens mais les méthodologies bactériologiques, en l'absence de cours pratiques, demeuraient assez mal connues des praticiens et des professeurs de médecine. Il y avait bien ça et là quelques leçons prodiguées par certains médecins intéressés par les développements de la bactériologie, tels le docteur Brennan, professeur à la succursale de Laval à Montréal qui installe en 1886 à l'Hôpital Notre-Dame un petit laboratoire de microscopie, de pathologie, de bactériologie et de chimie «muni de tous les appareils nécessaires pour procurer aux élèves des études cliniques pratiques en rapport avec les malades qu'ils voient journellement ${ }^{5} \gg$. Mais de telles leçons ne pouvaient guère offrir une formation adéquate pour permettre la transmission du savoir bactériologique au sein de la profession médicale.

Au tournant de la décennie 1890 , les jeunes médecins intéressés par cette nouvelle discipline se voient donc contraints de s'exiler pour un temps en Europe où sont développés à Paris et à Berlin des cours théoriques et pratiques de «microbie ${ }^{6} »$. Ces jeunes médecins francophones et anglophones deviendront en quelque sorte, selon l'expression de Claire Salomon-Bayet, les hommes-relais de la bactériologie en terre québécoise ${ }^{7}$. Ils ne tarderont pas à s'intégrer à l'élite acadé-

4. Pour un bref aperçu de l'introduction de la bactériologie au Québec, voir A. Frappier, Un rêve, une lutte. Autobiographie (Sillery, Presses de l'Université du Québec, 1992); L. Chartrand, R. Duchesne et Y. Gingras, Histoire des sciences au Québec (Montréal, Boréal, 1987), 340ss.; J. Bernier, La médecine au Québec, naissance et évolution d'une profession (Québec, Les Presses de l'Université Laval, 1989), 148-153; C.-M. Boissonnault, Histoire de la faculté de médecine de Laval (Québec, Presses Universitaires Laval, 1953), 301ss.

5. Rapport annuel de l'Hôpital Notre-Dame (1887-88): 15.

6. L'un des précurseurs semble avoir été Alfred Simard qui, entre les années 1860-1864, a suivi des cours de Claude Bernard et de Louis Pasteur. Voir C.-M. Boissonnault, Histoire de la faculté de médecine de Laval (Québec, Presses universitaires Laval, 1953), 279.

7. C. Salomon-Bayet, dir., Pasteur et la révolution pastorienne (Paris, Payot, 1986). En ce qui regarde les États-Unis, Rothstein souligne que «to teach the laboratory courses, the medical schools turned to the young European-trained medical scientists, who were the only ones trained in microscopy and laboratory methods. Many medicals schools turned over the entire basic science curriculum to one or two individuals», American Medical Schools and the Practice of American Medicine (New York and Oxford, Oxford University Press, 1987), 106. 
mique et médicale de la première décennie de notre siècle et à promouvoir le développement, selon leurs propres termes, d'une médecine «scientifique ${ }^{8} »$.

\section{LES HOMMES-RELAIS DE LA BACTÉRIOLOGIE EN TERRITOIRE QUÉBÉCOIS}

L'orientation médicale que prenaient les recherches bactériologiques durant la décennie 1880 rendait de plus en plus nécessaire la mise sur pied d'un enseignement théorique et technique pour initier les praticiens à ces nouvelles pratiques. Or, les facultés de médecine avaient tendance en Europe comme en Amérique à ignorer un tel enseignement ${ }^{9}$. Lors de l'inauguration de l'Institut Pasteur, le 18 novembre 1888, Pasteur soulignait les trois fonctions essentielles qui incombaient à cette nouvelle institution: «[II] sera à la fois un dispensaire pour le traitement de la rage, un centre de recherche pour les maladies infectieuses et un centre d'enseignement pour les études qui relèvent de la microbie ${ }^{10}$.»

Dès le 15 mars 1889 , le proche collaborateur de Pasteur, le docteur Émile Roux, se voit confier l'inauguration du premier cours de microbie technique. Fait significatif, Roux décide d'orienter son enseignement sur «les méthodes microbiennes dans leur application à la médecine». Essentiellement basé sur la pratique des méthodes microbiennes, l'enseignement de Roux visait à faire connaître aux élèves «les organismes microscopiques les plus intéressants, surtout ceux qui sont pathogènes, et les mettre à même de faire des travaux personnels ${ }^{11} \gg$. Un tel choix n'est pas anodin si on considère l'importance que prendront les techniques bactériologiques dans la pratique médicale et l'appui qu'apportera ultérieurement la profession médicale à la promotion de cette nouvelle discipline. Une telle orientation favorisait

8. L'emploi fréquent de cette expression par les Parizeau, Dubé, Mercier, etc., est fort mal à propos puisque la médecine québécoise était, depuis au moins la deuxième moitié du XIX ${ }^{e}$ siècle, une médecine basée sur une approche clinique qui suivait les enseignements des Écoles de Paris, de Londres, de Berlin ou d'Édimbourg et dont les pratiques s'appuyaient en partie sur les acquis de la physiologie expérimentale à partir des travaux de Magendie, Bernard, Traube ou Müller. On peut comprendre néanmoins que les nouvelles méthodes diagnostiques et thérapeutiques issues de travaux en laboratoire soient apparues aux yeux de certains comme les fondements d'une nouvelle médecine «scientifique».

9. «Peu importe que les facultés de médecine soient inertes et ne modifient pas, ou peu, leur enseignement: le microbiologiste, comme le microbe, se multiplie dans les milieux propices», C. Salomon-Bayet, op. cit., 50.

10. M. Faure, Histoire des cours de l'Institut Pasteur (Institut Pasteur, n.d.), 3. Sur l'histoire de l'Institut Pasteur voir M. Morange, dir., L'Institut Pasteur. Contributions à son histoire (Paris, Éditions La Découverte, 1991).

11. Archives de l'Institut Pasteur, Paris, document 9474. 
une clientèle surtout composée de médecins susceptibles de répandre parmi leurs collègues les habitudes de laboratoire. Le premier cours, prodigué dans le laboratoire de l'Institut, accueille un groupe de 15 élèves composés de sept internes des hôpitaux et de six médecins. La durée d'un tel cours variera à ses débuts entre cinq et six semaines ${ }^{12}$. Peu à peu les sessions d'études s'allongeront et en 1894, elles comporteront 45 leçons échelonnées sur huit semaines, leçons prodiguées principalement jusqu'en 1902 par Roux et Metchnikoff.

\section{Les médecins québécois inscrits au «cours de microbie technique» de l'Institut Pasteur}

Les registres de l'Institut Pasteur nous indiquent que plusieurs médecins québécois suivent, durant les décennies 1890 et 1900, le cours de microbie technique du docteur Roux. Ceux-ci ont toutefois eu quelques prédécesseurs. Les docteurs Rodier et McConnell furent les premiers médecins québécois à s'initier sur place aux techniques bactériologiques lorsqu'ils obtinrent en 1886 la permission de suivre les travaux de Pasteur à son laboratoire au 45 rue d'Ulm à l'École Normale de Paris. À la fin novembre 1888, le docteur A. Hamel, se rend, en tant que représentant de la faculté de médecine de l'Université Laval, à l'Institut Pasteur nouvellement inauguré ${ }^{13}$. Pasteur lui accorde dès son arrivée l'autorisation de visiter l'Institut ${ }^{14}$. Il aurait alors suivi quelques leçons du docteur Roux portant sur les méthodes bactériologiques appliquées à la médecine ${ }^{15}$. Dès son retour à la faculté de médecine de l'Université Laval à la mi-février 1889, il prodigue quelques leçons théoriques rudimentaires de «microbie» dans le cadre de son cours d'histologie pathologique.

La première inscription officielle d'un médecin québécois au cours de microbie technique de l'Institut Pasteur a lieu au printemps

12. Le formulaire d'inscription indique que «les personnes qui désirent suivre le cours doivent se faire inscrire à l'économat de l'Institut Pasteur (25, rue Dotot); elles doivent payer un droit d'inscription de 50 francs». Archives de l'Institut Pasteur, document 9474.

13. Le départ du docteur Hamel, le 14 novembre 1888, précède de peu l'inauguration de l'Institut Pasteur qui a lieu quatre jours plus tard. «Le docteur Auguste Hamel part aujourd'hui pour New York, où il prendra passage à bord de la Gascogne, pour le Havre. Notre distingué et laborieux concitoyen va passer une couple de mois à Paris, pour y faire des études spéciales, sous la direction des maîtres en son art: l'histologie et la bactériologie. Le docteur Hamel fait le voyage en compagnie de $M$. l'abbé Laflamme, le savant sécrétaire de l'université Laval», L'Événement, 14 novembre 1888.

14. Pasteur le reçoit sur la rue d'Ulm en décembre 1888 et lui délivre un certificat de visite qui porte la mention suivante: «M. Damiot voudra bien faire visiter l'Institut Pasteur à $\mathrm{M}$. le docteur Hamel, de l'université de Québec (Canada)». Carte et document de la famille du docteur Hamel, reproduit dans le Laval Médical (septembre 1957): 190.

15. Il s'agit probablement des premières leçons données par Roux puisque celui-ci ne débute son «cours de microbie technique» qu'au printemps 1889. 
1890. Le docteur Brodeur de Montréal suivra un cours intensif échelonné entre le 2 mai et le 5 juillet ${ }^{16}$. Deux ans plus tard, le docteur A. Marien assiste à un cours spécifique donnée par Weinberg sur les agents anaérobiques responsables des infections putrides ${ }^{17}$. Il s'inscrira aussi, peu avant son retour à l'Hôtel-Dieu de Montréal en 1895, au «Cours supérieur de bactériologie de l'Institut Pasteur ${ }^{18}$ ». De retour à Paris l'année suivante, il s'incrit à la session d'été au cours de microbie technique toujours dirigé par Roux et Metchnikoff. Entre-temps, les docteurs Larue et Laberge, respectivement en 1892 et 1893, s'y étaient inscrits. Le docteur Laberge deviendra peu après bactériologiste de la ville de Montréal.

C'est durant la période comprise entre les années 1896 et 1905 que la représentation québécoise à l'Institut Pasteur sera la plus forte avec vingt médecins québécois inscrits. Ceci correspond d'ailleurs, nous le verrons plus loin, à l'augmentation considérable de l'intérêt manifesté par les médecins à la suite de la présentation du sérum antidiphtérique par Roux à Budapest. En 1896, cinq jeunes médecins québécois - les docteurs Benoît, Parizeau, Marien, Mercier et Lasnier - figurent sur la liste des inscrits des docteurs Roux et Metchnikoff. L'année suivante, le trifluvien G. Bourgeois fait partie des 49 étudiants inscrits aux leçons de microbie technique. Marien, Mercier et Bourgeois se consacreront surtout à introduire les applications chirurgicales qui découlent des thèses bactériologiques. Le docteur T. Parizeau réorganisera, au retour de son stage à l'Institut Pasteur en 1896, les laboratoires de bactériologie et de pathologie de la faculté de médecine de la succursale de l'Université Laval à Montréal. Son assistant nommé la même année, le docteur W. Derome, avait lui aussi effectué un séjour à l'Institut Pasteur. Il publiera dans L'Union médicale du Canada ses notes de cours où il rend compte des travaux de Kitasato, Faber, Berhing, Metchnikoff, etc. ${ }^{19}$ Parizeau sera nommé professeur de bactériologie en 1900. Après avoir passé un an

16. Exceptionnellement, c'est le docteur Yersin qui remplace le docteur Roux durant cette session. Il est à noter que sur les 14 inscriptions, il n'y a que deux étrangers. Archives de l'Institut Pasteur, Cours de microbie technique 1889-1956, Liste des élèves, 5.

17. À son retour, Marien se consacrera exclusivement à la pratique chirurgicale et il introduira, vers 1895, l'asepsie opératoire à l'Hôtel-Dieu de Montréal.

18. Archives de l'Institut Pasteur, Liste des inscrits au «Cours supérieur de bactériologie de l'Institut Pasteur».

19. Voir par exemple, W. Derome, «Le tétanos. Cours de bactériologie. - Quarante deuxième leçon. Notes recueillies à l'Institut Pasteur», L'Union médicale du Canada (1898): 207-217. Derome retournera à Paris en 1908 pour se spécialiser en anatomie-pathologique et devient médecin légiste de l'Université de Paris. À son retour en 1909, il reprend son travail au laboratoire d'anatomie-pathologique et se voit confier la tâche de constituer «une collection de spécimens pathologiques». Il deviendra aussi en 1912 directeur du laboratoire de l'Institut Bruchési. Cf. Rapport Annuel de l'Hôpital Notre-Dame (1909), 25. 
sous la tutelle des bactériologistes Roux et Martin en 1898, le docteur A. Bernier deviendra à son retour, l'année suivante, démonstrateur de bactériologie et, en 1899, chef du laboratoire de bactériologie de l'Hôpital Notre-Dame. Il sera aussi nommé bactériologiste en chef du Conseil d'hygiène de la province de Québec en 1908 et deviendra le premier professeur titulaire de bactériologie à la faculté de médecine de la succursale de 1'Université Laval à Montréal en $1910^{20}$, postes qu'il conservera jusqu'en 1928. Il cumulera aussi les fonctions de directeur du laboratoire de l'Hôpital Notre-Dame et de bactériologisteconsultant de l'Hôtel-Dieu.

En juin 1899, le docteur A. Rousseau revient de Paris «avec toute une armée de microbes, choisis parmi les plus étranges et les plus meurtriers ${ }^{21}$ » annonce le recteur de l'Université Laval. À la fin du $\mathrm{XIX}^{\mathrm{e}}$ siècle, les docteurs J.-E. Dubé et A. Lesage suivent tous deux les cours du clinicien Dieulafoy et du bactériologiste Widal. En 1900, le docteur C.-A. Daigle, après avoir effectué des travaux de pathologie avec le bactériologiste A. Bruère au Royal Victoria Hospital en 18951897, décide d'aller se perfectionner à Paris. Il y suit les cours du professeur Letulle et exécute des travaux de laboratoire sous la direction du bactériologiste Weinberg. À son retour en 1901, il sera nommé démonstrateur de bactériologie et d'anatomie-pathologique à la faculté de médecine de la succursale de l'Université Laval à Montréal. La même année, il sera chargé d'organiser un laboratoire d'analyse et de bactériologie pour le service de la maternité à l'Hôpital de la Miséricorde. Le docteur Arthur Vallée, après avoir effectué un stage à l'Institut Pasteur et suivi les travaux de Metchnikoff, prend la relève de Rousseau en 1907 et organise un nouveau laboratoire de bactériologie à l'Université Laval.

Pour la première fois en 1901-1902, cinq médecins québécois les docteurs Ethier, Larue, Tourigny, Mayrand et Bédard - suivent ensemble les sessions de cours de l'Institut Pasteur qui s'étendent de novembre à février. Tous deviendront de fervents défenseurs de la bactériologie en territoire québécois. Mentionnons à titre d'exemple, le docteur Bédard qui fondera en 1901 La revue scientifique, revue qui

20. Procès-verbaux de l'École de médecine et de chirurgie/Faculté de médecine de l'Université Laval à Montréal, 7 mars 1910, 53.

21. Boissonnault, op. cit., 276-277. Le docteur Rousseau s'était embarqué pour Paris le 18 février 1895 . Il consacre ses avant-midi à des stages de médecine interne dans les hôpitaux mais profite de son temps libre l'après-midi pour suivre les cours donnés par Roux, Duclaux et Metchnikoff. Nous n'avons toutefois trouvé aucune trace de son inscription à l'Institut Pasteur mais les registres sont à cet égard peu fiables surtout en ce qui regarde les auditeurs. Du reste, La Revue médicale publiera des extraits du cours d'Émile Roux rédigé par Rousseau et daté du 4 janvier 1896. 
avait pour but de «répandre parmi le peuple les principes d'hygiène moderne» et qui sera l'auteur en 1898 d'une brochure intitulée Les maladies infectieuses: diagnostic bactériologique, sérothérapie.

Contrairement aux médecins francophones qui se rendaient généralement à l'Institut Pasteur pour se perfectionner en bactériologie, les médecins anglophones choisissaient, pour des raisons culturelles et linguistiques, des stages dans les centres germaniques et britanniques reconnus pour leurs travaux en pathologie. Le docteur W. G. Johnston, au tournant des années 1890, séjourne en Allemagne dans les laboratoires de Virchow et de Growitz où il se consacre à l'étude de la bactériologie. C'est là qu'il puise les connaissances fondamentales qui lui permettront d'enseigner et de pratiquer la bactériologie à l'Université McGill et au Montreal General Hospital. Il deviendra l'un des premiers Canadiens à joindre à ses débuts la Society of American Bacteriologists $^{22}$. L'assistant de Johnston à McGill, C.-F. Martin, avait étudié la pathologie et la bactériologie en Grande-Bretagne et en Allemagne. Le docteur A. MacPhail, professeur de pathologie et de bactériologie à la Bishop's Medical faculty, avait suivi des cours postgradués à Londres durant les années 1890 . H. Oertel, engagé comme professeur de pathologie à l'Université McGill en 1914, avait fait des études post-gradués à l'Institut de pathologie de Würzburg de même qu'à Leipzig. Exceptions notables, les docteurs McConnell et Adami se sont perfectionnés en bactériologie à Paris. À la session d'automne 1890 , le docteur G. Adami, alors démonstrateur d'anatomie pathologique à l'Université de Cambridge, s'inscrit au cours du docteur Roux à l'Institut Pasteur. Mentionnons qu'Adami occupera peu après la chaire de pathologie et de bactériologie à l'Université McGill.

Les notes de cours manuscrites consultées à l'Institut Pasteur ${ }^{23}$ s'avèrent précieuses pour mesurer le bagage théorique et pratique que rapportaient ces jeunes médecins. Les quarante-cinq leçons prodiguées par Roux et Metchnikoff à partir de 1894 constituent un éventail de règles théoriques et pratiques qui illustrent dans le détail les rigoureux procédés bactériologiques auxquels étaient initiés les étudiants inscrits: présentation de la théories des germes opposée à la génération spontanée; classification, morphologie et action des microbes; cultures sur gélatine; examen, coloration et séparation des microbes; stérilisation des infusions organiques, etc. À cela s'ajoutaient les techniques d'analyse microbienne de l'air, des eaux et du sol; les techniques de

22. D. Goulet et O. Keel, «W. G. Johnston», Dictionnaire biographique du Canada (Québec et Toronto, Presses de l'Université Laval/University of Toronto Press), XIII: à paraître.

23. Archives de l'Institut Pasteur, Cours de microbie technique 1890-1900. Soulignons que le contenu du cours variera assez peu durant cette période. 
culture des microbes anaérobiques; l'apprentissage des inoculations expérimentales, la recherche du bacille sur l'animal vivant ou l'autopsie d'animaux infectés ainsi qu'une série de leçons sur les caractères bactériologiques et les modes de propagation des principales maladies infectieuses: charbon, rage, pneumonie, fièvre typhoïde, tuberculose, choléra, diphtérie, tétanos, etc. Une série de leçons portaient plus spécifiquement sur les phénomènes de suppuration et sur les microbes pyogènes. Le stagiaire, à la suite d'un exposé où était démontré qu'il n'y a pas de «pus sans microbes», procédait à l'examen du pus et à la recherche des staphylocoques et des streptocoques. On imagine l'intérêt que devait susciter cette matière chez les chirurgiens. Étaient ensuite exposés les procédés de désinfection par les moyens physiques - eau bouillante, chaleur sèche, chaleur humide et étuves à vapeur et les moyens chimiques - antiseptiques divers. Soulignons enfin que les étudiants avaient l'occasion de s'initier aux première données de l'immunologie - virulence, phagocytose, etc. - par l'un de ses principaux pionniers, Metchnikoff.

Les médecins québécois inscrits à ces leçons ont donc reçu une formation théorique et pratique adéquate des techniques microbiennes qui leur permettra d'enseigner et d'appliquer les techniques bactériologiques en sol québécois. Ils contribueront largement au tournant $\mathrm{du} \mathrm{XX}^{\mathrm{e}}$ siècle, par leur introduction au sein des structures médicales institutionnelles - revues, universités, sociétés médicales et hôpitaux - à l'émergence d'une médecine bactériologique orientée vers le laboratoire et donneront, malgré les obstacles financiers, idéologiques et épistémologiques, une nouvelle direction à certaines pratiques chirurgicales et hygiéniques. Mais il faut se garder d'y voir le triomphe radical des modernes contre les anciens ou d'y rechercher une rupture fondamentale. Car c'est bien de transition qu'il est question ici et si épistémologiquement et méthodologiquement la doctrine microbienne et les techniques bactériologiques constituaient un acquis majeur, elles ne transforment pas du jour au lendemain l'enseignement, les pratiques et les représentations de la profession médicale. Il fallait en un premier temps assurer la transmission et la reproduction des techniques bactériologiques en territoire québécois.

Marien et ses jeunes confrères québécois de l'Institut Pasteur qui, se réunissaient depuis 1896 pour discuter des nouveautés médicales, seront à l'origine de la renaissance de la Société médicale de Montréal quatre ans plus tard. Une telle initiative permettait la promotion des nouveaux acquis de la science médicale. De nombreux mémoires de la société porteront notamment sur les développements de la bactériologie et des méthodes antiseptiques et aseptiques. Par ailleurs, Marien, Lesage, Harwood, Boulet et Dubé font l'acquisition en 1900 
de la principale revue médicale francophone du Québec, L'Union médicale du Canada, acquisition qui leur permettra de «travailler à [l']avancement scientifique en répandant par tout le pays les idées nouvelles qui germent dans les centres intellectuels du monde entier ${ }^{24} \gg$. La mainmise de ces jeunes pastoriens sur ces deux institutions indique bien que ces réformateurs entendaient jouer un rôle important dans l'orientation scientifique de la profession médicale.

\section{LES PREMIERS COURS DE BACTÉRIOLOGIE EN TERRITOIRE QUÉBÉCOIS}

Dès les années 1880 , certains médecins avaient obtenu la possibilité de prodiguer quelques leçons de bactériologie mais il ne s'agissait généralement que d'un enseignement théorique ponctuel et irrégulier. C'est véritablement à partir de la décennie 1890, grâce à diverses initiatives prises par les autorités médicales, que se mettent en place les prémisses d'un enseignement structuré de la bactériologie en territoire québécois.

Le docteur Hamel, professeur à la faculté de médecine de l'Université Laval à Québec, introduit en 1889 quelques leçons théoriques de bactériologie au sein de son cours d'histologie pathologique et est promu, durant la session 1893-1894, professeur titulaire du nouveau cours de bactériologie théorique et d'histologie pathologique $^{25}$. En 1890, la faculté de médecine du Bishop's College met sur pied des laboratoires d'histologie et de bactériologie ${ }^{26}$. L'année suivante, l'Université McGill décide de confier au docteur W. G. Johnston, déjà démonstrateur de pathologie depuis 1885 , l'un des premiers, sinon le premier poste de lecturer en bactériologie en territoire canadien. Johnston est alors assisté du docteur C.-F. Martin. Si depuis 1885, la faculté de médecine de McGill offrait à sa clientèle un laboratoire de pathologie contenant «all instruments and apparatus necessary for the more advanced student graduates and graduates who wish to engage in private pathological research ${ }^{27}$ », c'est à partir de 1890, que l'organisation du laboratoire de pathologie au sein de la faculté accorde davantage d'espace et d'équipements pour les travaux

24. L'Union médicale du Canada (1900), 324.

25. Annuaire de l'Université Laval (1893-1894): 122. L'enseignement de Hamel, strictement d'ordre théorique, s'attarde surtout sur les agents de suppuration tels que les staphylocoques, les gonocoques et les méningocoques. Sont aussi étudiés, le bacille de la typhoïde, du charbon et de la tuberculose. Le docteur Hamel deviendra en 1902 professeur d'histologie normale et pathologique et de bactériologie théorique et ce, jusqu'à la session 1922-1923. Annuaire de l'Université Laval (1922-1923).

26. E. Hearn Milner, Bishop's Medical Faculty Montreal 1871-1905 (Sherbrooke, R. Prince Imp. Inc., 1985), 453.

27. McGill University Calendar (1885-1886), 84. 
bactériologiques. Il comprend alors un ensemble «of rooms exclusively devoted to the study and culture of Bacteria, furnished with a complete outfit of the best modern apparatus for this purpose, including sterilizer, thermostat, etc. ${ }^{28}{ }_{\nu}$ L'ouverture du Pathological Building en 1895 permettra une amélioration notable des équipements de laboratoire.

Il faut attendre l'année 1894 pour que soit rendu obligatoire l'enseignement de la bactériologie dans les facultés médicales du Québec ${ }^{29}$. Les arrêtés proposés par le Bureau médical de la province et ratifiés par le Gouverneur en Conseil les 4 janvier 1894 et 28 octobre 1896 ajoutent au curriculum plusieurs cours spéciaux dont un cours d'histologie pathologique et de bactériologie mais laissent indéterminé le nombre de leçons à prodiguer. Les universités conservaient toutefois l'opportunité de décider le rapport des cours théoriques et des séances de laboratoire de sorte que, jusqu'aux années 1900 , la bactériologie ne constituera généralement pas un cours autonome mais demeurera subordonné à l'enseignement de la pathologie ${ }^{30}$ et de l'histologie. Du reste, les quelques cours prodigués demeureront le plus souvent essentiellement théoriques.

Pour répondre à cette nouvelle obligation, l'École de médecine et de chirurgie/Faculté de médecine de l'Université Laval à Montréal ajoute 60 leçons d'histologie normale et de bactériologie élémentaire au curriculum des étudiants de première année ainsi que des cours de bactériologie pratique aux étudiants de troisième et quatrième années $^{31}$. La faculté confie en 1898 le cours d'anatomie-pathologique et de bactériologie - qui était jusque-là sous la responsabilité de Brennan - à T. Parizeau, poste qu'il occupera jusqu'en 1902. Quant à la faculté de médecine de l'Université Bishop, elle confie en 1899 le cours de bactériologie au docteur J. B. McConnell. Il sera remplacé l'année suivante par le docteur A. MacPhail.

28. McGill University Calendar (1890-1891), 89.

29. C'est en 1891 que le Medical Council of Ontario instaure l'enseignement obligatoire de la bactériologie et de la pathologie. La faculté de médecine de l'Université Queen's consacrait en 1891 deux heures par semaine d'enseignement de la bactériologie et le premier professeur titulaire de pathologie et de bactériologie, le docteur W. T. Connell, est nommé en 1895 . Ce dernier publiera en 1899, son Practical Bacteriology. Cf. A. A. Travill, Medicine at Queen's 1854-1920 (Faculty of Medicine, Queen's University, The Hannah Institute for the History of Medicine, 1988), 111, 167-168.

30. Il en est de même aux États-Unis: «...bacteriology was as yet (circa 1900) not a fully separate discipline. It was, in fact, still largely a subdomain of pathology.» R. C. Maulitz, «Physician versus bacteriologist», M. J. Vogel et C. E. Rosenberg, eds., The Therapeutic Revolution: Essays in the Social History of American Medicine (University of Pennsylvania Press, 1979), 93.

31. Voir à ce propos, les Annuaires de l'École de médecine et de chirurgie/Faculté de médecine de l'Université Laval à Montréal (1894-1910). 
La faculté de médecine de l'Université Laval officialise en 1894 les leçons de bactériologie dispensées par Hamel au sein de son cours d'histologie pathologique et organise, en attendant le retour d'Arthur Rousseau prévu pour la fin juin 1899 , un petit laboratoire pratique de bactériologie «à l'usage des élèves»:

les microbes, tout menus qu'ils soient [déclare le recteur], deviennent des personnages si importants, que l'Université a jugé à propos de mettre les élèves à même de faire plus ample connaissance avec eux. Les travaux que nos étudiants feront dans ce nouveau laboratoire leur montreront ces microscopiques mais innombrables ennemis. À eux de trouver comment nous en débarrasser $^{32}$.

En 1899, le Bureau médical de Québec propose d'amender les règlements de 1896 et d'accorder un minimum de 20 heures de leçons théoriques et 60 heures de leçons pratiques. Mais de vives oppositions retardent la mise en œuvre d'un tel programme. L'un des membres du comité chargé d'étudier le programme des études médicales s'y objecte:

on attache une trop grande importance dans le rapport de votre comité aux travaux de laboratoire, qui quoiqu'utiles, ne doivent pas prendre une trop grande place dans l'enseignement [...] les études de laboratoire par la longueur du temps qu'elles exigent ne peuvent tendre qu'à former des savants spécialistes et non des médecins pratiquants ${ }^{33}$.

De telles objections ne sont pas rares et traduisent la difficulté d'ajuster les pratiques de laboratoire avec les pratiques cliniques. Néanmoins, à partir de 1900 , certains travaux pratiques de bactériologie sont rendus obligatoires pour les élèves de la faculté de médecine de Laval. L'enseignement de la bactériologie prend peu à peu davantage d'importance. En 1907, les facultés avaient été contraintes de se plier aux nouvelles exigences du Bureau de médecine de la province de Québec qui rendit obligatoire un cours de trois mois de bactériologie théorique et pratique, mesure qui en certains cas confirmait certaines initatives antérieures. Lorsque le docteur A. Bernier prend la relève de Parizeau à la faculté de médecine de Laval à Montréal en 1902, le curriculum des cours d'histologie/bactériologie et de bactériologie/anatomie-pathologique avait été augmenté à 120 leçons, chaque cours débouchant respectivement sur un examen primaire et sur un examen final. En 1910, toujours sous la gouverne

32. Annuaire de l'Université Laval (1898-1899), 143.

33. Bulletin médical de Québec (1900), 681. 
de Bernier, 120 leçons de bactériologie sont dispensées à la faculté ${ }^{34}$. Afin d'illustrer et d'expérimenter les effets des germes, les facultés médicales de Québec et de Montréal avaient mis sur pied de petites animaleries contenant des lapins et des cobayes. Selon le témoignage d'Armand Frappier, les cours de bactériologie prodigués par Bernier jusqu'à son décès survenu en 1929 , «représentaient à peu près l'équivalent de ceux qu'on donnait aux étudiants en médecine à l'Université de Rochester, New York ${ }^{35}$ ».

Néanmoins, l'organisation des laboratoires des facultés médicales francophones de Québec et de Montréal laissait à désirer. Bien des membres de ces facultés n'accordaient alors que peu de crédits aux travaux pratiques de laboratoire et ne partageaient guère l'enthousiasme des jeunes médecins «pour les nouvelles théories microbiennes». Or, les membres du conseil des facultés avaient un pouvoir quasi absolu sur l'organisation didactique et «scientifique» de leur faculté. Les moyens accordés pour l'organisation des laboratoires demeuraient parfois à la mesure du scepticisme des dirigeants. Une telle situation n'était pas exclusive au Québec. Flexner dans son célèbre rapport en 1910 sur l'enseignement médical aux États-Unis et au Canada ${ }^{36}$ souligne le mauvais état général des sections de bactériologie dans de nombreuses facultés médicales des États-Unis et du Canada ${ }^{37}$.

En 1920, la Rockefeller Foundation offre aux facultés médicales du Canada un don global de $2 \$$ millions pour améliorer la formation médicale au Canada, notamment en ce qui regarde le développement de la médecine de laboratoire. À la suite d'une visite de son délégué à la faculté de médecine de l'Université de Montréal, le docteur Pearce, elle exige, comme condition d'obtention d'une subvention,

34. En 1911, les docteurs Bernier et Aubry sont respectivement chargés des cours de bactériologie théorique et de bactériologie pratique. Trois ans plus tard, le docteur Bernier décide, sous condition - acceptée par la faculté - que son salaire soit porté annuellement à $2000 \$$, de laisser son poste au laboratoire de l'Hôtel-Dieu de Montréal pour se consacrer à ses cours de bactériologie et de pathologie générale. Cf. Procès-verbaux de l'École de médecine et de chirurgie/Faculté de médecine de l'Université Laval à Montréal, 7 novembre 1911, 93, et 8 septembre 1914, 176.

35. A. Frappier, op. cit., 68. L'auteur ajoute que le professeur Bernier «comptait parmi les meilleurs bactériologistes du Canada».

36. Ce rapport est intitule Medical Education in the United States and Canada.

37. L'enseignement y laisse souvent à désirer: «there are certain men who teach bacteriology who start at the beginning of their lectures with a lot of tubes already made. They do not know enough about bacteriology to make cultures. They hold up these tubes and say, 'This is a diphteria culture; this is a culture of tubercle bacillus', and if by any chance a culture goes bad, they send it and get another [...] Bacteriology fares in general rather worse.» A. Flexner, op. cit., 85. L'auteur cite de nombreux exemples de laboratoire dont l'équipement et l'organisation sont rudimentaires. 
que la faculté instaure une année préparatoire d'études en science, qu'elle modernise son laboratoire et enfin qu'elle accorde une part plus importante aux travaux de laboratoire ${ }^{38}$ dans son curriculum. Deux ans plus tard, le rapport du docteur Pearce juge satisfaisants les locaux attribués aux laboratoires ${ }^{39}$ et les corrections apportées à la formation pratique des étudiants. Chaque étudiant reçoit désormais une formation individuelle en laboratoire, particulièrement en bactériologie. Un second rapport daté du 21 juin 1923 souligne l'apport de Harwood qui est jugé comme «a progressive dean, with power, who is willing to be taught modern views of medical education ${ }^{40}{ }_{\nu}$. Malheureusement des difficultés majeures - incendie, crise économique retarderont le développement scientifique de la faculté. Les laboratoires de la faculté de médecine de Québec et de ses hôpitaux affiliés ont connu un développement à peu près similaire à celui de sa consœur de Montréal. Quant aux laboratoires de pathologie de la faculté de médecine de l'Université McGill, ils pouvaient avantageusement se comparer aux meilleures institutions du continent.

Les initiatives mentionnées ci-haut en matière d'enseignement de la bactériologie ont été largement favorisées par les jeunes étudiants québécois diplômés de l'Institut Pasteur ou des centres de pathologie en Allemagne ou en Autriche. Les chargés de cours, assistants ou démonstrateurs en bactériologie, histologie, pathologie ou en chirurgie ont pour la plupart été sélectionnés parmi ceux-ci. Ils s'efforceront d'enseigner les applications diagnostiques, prophylactiques et thérapeutiques de la bactériologie. Quant à ceux qui obtiendront des charges de cours en chirurgie, ils enseigneront certaines modifications du protocole opératoire découlant des thèses bactériologiques. Leur pénétration au sein des facultés de médecine a parfois été facilitée par l'intercession de doyens ou de membres des conseils qui reconnaissaient le bien-fondé de cet enseignement. Tel est le cas du docteur E.-P. Lachapelle qui, nommé en 1910 doyen de l'École de médecine et de chirurgie/Faculté de médecine de l'Université Laval à Montréal en remplacement de Rottot, facilitera la reconnaissance de la médecine pasteurienne. Ses successeurs, anciens stagiaires à Paris, poursuivront en ce sens. Le doyen de Lotbinière Harwood appuiera en 1925 la mise en œuvre du projet de recherche clinique du docteur Beaudoin sur le BCG. Mentionnons aussi que le doyen T. Parizeau

38. The Rockefeller Foundation Archives, Université de Montréal, Faculté de médecine, 1919-1920, 1.

39. Pearce mentionne l'année suivante: «The Faculty at Montreal [...] allows a rearrangement of laboratory space on a modern basis.» The Rockefeller Foundation Archives, Université de Montréal, Faculté de médecine, 1923, 21.

40. Ibid., 22. 
encouragera le docteur Frappier, au début des années 1930, à entreprendre des études en microbiologie ${ }^{41}$.

L'enseignement de la bactériologie ne se confinait cependant pas aux seules facultés médicales. Ses aspects prophylactiques, diagnostiques et thérapeutiques intéressaient aussi les secteurs connexes du champ médical, tels que l'hygiène publique, la médecine vétérinaire ou l'art dentaire. Durant les années 1910, la bactériologie, avec la chimie, occupe une place très importante dans le cursus du diplôme en hygiène et santé publique décerné par les trois facultés (McGill, Laval et Montréal $)^{42}$. Desrosier et al. montrent que, vers 1914-1915, les cours de bactériologie et de chimie occupent à McGill, 9 des 15 mois d'études, alors qu'à Montréal, ils comptent pour 58 des 128 leçons prodiguées contre $60 / 190$ pour Laval ${ }^{43}$. Écoles vétérinaires, écoles d'infirmières, écoles de pharmacie, laboratoires d'hygiène publique ou instituts vaccinaux dispensent aussi quelques cours de bactériologie. Sont enseignés, dans les écoles de pharmacie, la préparation des sérums et l'utilisation des tests sérologiques. En 1926, l'École de médecine vétérinaire de l'Université de Montréal met sur pied un laboratoire de bactériologie ${ }^{44}$ pour y enseigner les procédés de tuberculinisation des vaches laitières. Pendant que les infirmières sont initiées aux théories bactériologiques et aux procédés de désinfection, des cours polycliniques qui abordent différents aspects de la bactériologie sont offerts aux médecins de l'Hôpital Notre-Dame désireux de se perfectionner. En 1913, à la suite des pressions exercées par les médecins hygiénistes de Québec, un cours clinique sur les maladies contagieuses est mis sur pied à l'Hôpital civique de Québec. Il fallait pour que les laboratoires provinciaux et municipaux fonctionnent efficacement dans le diagnostic et la thérapeutique de certaines maladies contagieuses, promouvoir l'utilisation des techniques bactériologiques dans la pratique clinique ${ }^{45}$.

\section{LA PRATIQUE CLINIQUE ET LES NOUVELLES MÉTHODES BACTÉRIOLOGIQUES}

La transition d'une médecine hospitalière vers une médecine de laboratoire s'est effectuée lentement et la plupart des praticiens

41. A. Frappier, op. cit., 61.

42. Voir G. Desrosiers, B. Gaumer, O. Keel, «L'enseignement universitaire spécialisé de santé publique au Québec», Bulletin canadien d'histoire de la médecine/Canadian Bulletin of Medical History, 7,1 (1990): 7.

43. Ibid.

44. M. Pépin, Histoire et petites histoires des vétérinaires du Québec (Montréal, Éditions François Lubrina, 1986), 145.

45. Les analyses bactériologiques des laboratoires du Conseil d'hygiène se chiffrent à 296 en 1908, 551 en 1909, 1454 en 1910, 4610 en 1919, 57232 en 1928 et 80770 en 1930. 
préféraient s'en tenir encore durant la décennie 1890 au raffinement des procédés classiques d'investigation clinique et aux rares analyses de laboratoire que constituaient le dosage de l'urée et la recherche du taux d'albumine. Il faut dire que les cliniciens canadiens en général, contrairement aux cliniciens allemands, s'intéressaient assez peu aux démarches scientifiques exercées en laboratoire. La pratique clinique et chirurgicale était essentiellement perçue comme une praxis et dans la plupart des cas, les innovations diagnostiques ou thérapeutiques étaient jaugées en terme d'efficacité. Or, à partir des années 1890 , l'impact qu'auront les découvertes bactériologiques, notamment du sérum antidiphtérique en 1894, sur la pratique clinique est considérable. On peut en mesurer l'effet sur la profession médicale à partir de l'intérêt que susciteront durant les années suivantes les leçons bactériologiques prodiguées à l'Institut Pasteur. Aussi, le rédacteur des registres des cours de l'Institut en 1896 prend-il la peine de souligner que: «Le nombre des auditeurs a de beaucoup dépassé celui des cours précédents. Il a varié entre 105 et 60 . Il comprenait beaucoup de médecins étrangers venus se mettre au courant de la sérothérapie de la diphtérie ${ }^{46} . »$

Roux et Metchnikoff avaient alors ajouté une leçon sur «les techniques de préparation de tubes de sérum coaguléc ${ }^{47} »$. D'autres recherches bactériologiques entreprises dans les laboratoires français et allemands s'étaient imposées aux praticiens sur le plan diagnostic et thérapeutique. Behring et Kitasato proposent en 1890 le traitement sérothérapique du tétanos, Widal et Gruber mettent au point le sérodiagnostic de la fièvre typhoïde en 1897 , etc. Soulignons aussi que grâce aux recherches effectuées à l'Institut sérothérapique de Paris entre 1894 et 1900, «les sérums antitétaniques, antistreptococciques, antipesteux sont entrés dans la pratique, distribués ou vendus ${ }^{48} \gg$. En 1906, est mis au point le test Bordet-Wassermann qui permet le dépistage de la syphilis. À l'instigation de Cole, directeur du Johns Hopkins Hospital, se popularisa au sein de la communauté médicale l'idée d'une réunion des modèles anglais et germaniques où l'investigation clinique au lit du malade devait être complétée par des analyses de laboratoire. De telles découvertes contribueront donc à amorcer le déplacement, certes encore limité, du diagnostic et de la thérapeutique classiques des maladies contagieuses vers des procédés diagnostiques et thérapeutiques issus des laboratoires de bactériologie. Alors que

46. Archives de l'Institut Pasteur, Cours de microbie technique 1889-1956, Liste des élèves, 30.

47. Archives de l'Institut P'asteur, Cours de microbie technique (1896).

48. C. Salomon-Bayet, dir., Pasteur et la révolution pastorienne (Paris, Payot, 1986). 
près de $20 \%$ des patients hospitalisés souffraient de maladies contagieuses, on comprend que les analyses bactériologiques de ces maladies aient largement contribué à instaurer une pratique de laboratoire qui deviendra de plus en plus soutenue au sein des grandes institutions.

\section{De nouvelles pratiques diagnostiques et thérapeutiques}

Créé la même année que l'annonce de la mise au point du sérum antidiphtérique par Roux en 1894, le laboratoire provincial de bactériologie met en circulation le fameux sérum ${ }^{49}$. L'année suivante, le Conseil d'hygiène en distribue plus de 1500 bouteilles. Les hygiénistes et une large part de la profession médicale accueillent avec enthousiasme cette immunisation passive de la diphtérie ${ }^{50}$. Entre 1894 et 1896 , les articles et les comptes rendus dans les revues médicales sur les traitements et les modes de dépistage bactériologique abondent $^{51}$. Tests sérologiques, instructions pour l'emploi du sérum antidiphtérique, mises en garde contre les mauvais sérums, témoignages et expérimentations ponctuent les chroniques. Mais les besoins sont tels que certaines institutions ont peine à s'adapter à cette nouvelle pratique thérapeutique. Le gouvernement fédéral refuse d'accepter l'envoi par le service des postes des tubes de prélèvement. Le petit laboratoire du bureau de santé de la ville de Montréal dirigé par J.-E. Laberge mis sur pied lui aussi en 1897 n'aura que trois ans plus tard les équipements nécessaires pour effectuer le diagnostic bactériologique de la diphtérie ${ }^{52}$.

Pour pallier les besoins les plus immédiats, le laboratoire provincial est mis en 1896 à la disposition de la profession médicale qui peut y commander des examens diagnostiques ${ }^{53}$. Distribution du sérum antidiphtérique et analyses bactériologiques y deviennent alors

49. Le laboratoire est alors dirigé par W. G. Johnston assisté du docteur Ruttan qui y effectue les analyses cliniques. Un médecin de Québec, le docteur C. Verge atteint de diphtérie, se procure dès 1894 le sérum à l'Institut Pasteur de New York: «J'ai eu l'honneur d'expérimenter sur moi-même le premier à Québec la sérothérapie.» L'Union médicale du Canada (1895): 17.

50. Un article intitulé «Instructions pour l'emploi du sérum antidiphtérique» est publié dans le Journal d'hygiène populaire, 11 (1894-1895): 332-334.

51. Une quarantaine d'articles ou de comptes rendus paraissent dans les revues médicales entre 1894 et 1897 . Cf. A. Paradis, H. Naubert, avec la collaboration de D. Goulet, Recension bilbiographique: les maladies infectieuses dans les périodiques médicaux québécois du XIXe siècle (Trois-Rivières, Université du Québec à Trois-Rivières, 1988).

52. Voir M. Tétrault, L'état de santé des Montréalais de 1880 à 1914, mémoire de maîtrise (histoire), Université de Montréal, 1979, 137. En 1900, le laboratoire de la ville de Montréal effectue environ 1000 examens. En 1914, le nombre d'analyses grimpe à 3000 . Rapport de l'état sanitaire de la cité de Montréal (1900-1914).

53. D. Goulet et A. Paradis, op. cit., 464. 
des activités quotidiennes. L'Hôpital civique de Montréal avait mis le sérum antidiphétrique à la disposition des patients dès juin 1895 . Sur 179 patients ainsi traités seulement 19 décèdent de la diphtérie. Le progrès est patent. Mais trop souvent, se plaignent les médecins de l'hôpital, les patients sont acheminés à un stade avancé de la maladie. Néanmoins, le taux de mortalité occasionnée par la diphtérie commença une forte décroissance particulièrement dans les milieux où intervenaient les bureaux de santé ${ }^{54}$ et en partie, grâce aux analyses effectuées dans les laboratoires hospitaliers. Mais en ce qui regarde la pratique médicale en milieu rural, les progrès de la sérothérapie furent beaucoup plus lents. Dépistage et diagnostic inadéquats, mesures de protection insuffisantes et recours au médecin à un stade avancé de la maladie constituaient des obstacles à la prévention et au traitement de cette maladie. Les coûts du sérum étaient souvent prohibitifs pour les familles les plus pauvres et si certains praticiens l'offraient gratuitement, la plupart refusaient d'assumer une telle dépense et demandaient la mise sur pied d'organismes de santé pour prendre en charge sa distribution.

Les médecins profitent aussi d'un nouveau procédé diagnostic de la fièvre typhoïde, le sérodiagnostic de Widal, qui s'ajoute à l'observation clinique. En 1896, le Conseil d'hygiène se targue d'être «le premier conseil provincial» à faire gratuitement le séro-diagnostic de la fièvre typhoïde avec du sang desséchés5. Le docteur W. Johnston, directeur de la division des laboratoires du Conseil d'hygiène de la province de Québec avait lui aussi entrepris des études sur le procédé d'agglutination avec du sang séché de façon à rendre plus accessible le test diagnostic de la fièvre typhoïde. Au congrès annuel de l'American Public Health Association en septembre 1896, il présente les résultats de ses recherches qui sont bien accueillies. Devancé par des études similaires entreprises à l'Institut Pasteur par Widal, il ne peut cependant revendiquer la paternité de ce nouveau procédés6. Néanmoins, ses travaux permettent au Conseil d'hygiène de la province de Québec de mettre le séro-diagnostic de la fièvre typhoïde au service

54. Les proportions de décès par la diphtérie chutèrent au Québec de 650/1 million à 260/ 1 million entre les années 1902 et 1911. En 1928, la ville de Montréal lancera une grande campagne d'immunisation contre la diphtérie.

55. "Aussitôt que le diagnostic sera fait, - en général 2 hrs p. m. le lendemain de la réception de l'échantillon au Laboratoire, - un rapport écrit sera transmis au médecin, par la malle. On peut également obtenir un rapport par téléphone en appelant le No 454.» Cf. «Le sérodiagnostic de la fièvre typhoïde au Conseil d'hygiène de la Province», L'Union médicale du Canada (1896): 636-637.

56. Les 21 et 22 juin 1895, les docteur Adami et Johnston présentent à New York des communications au premier congrès consacré exclusivement à la bactériologie. 
de la profession médicale ${ }^{57}$ ce qui a contribué à répandre l'usage du sérodiagnostic chez les praticiens québécois. Le procédé demeure toutefois controversé: «on s'en prend à la méthode alors que c'est l'analyse qui manque de précision ou le médecin qui manque de persévérance ${ }^{58}$ ", note le docteur Lesage en 1902 qui insiste d'ailleurs pour que ses collègues ne négligent «aucun des moyens dont disposent la clinique et le laboratoire pour éclairer ou même raffermir un diagnostic dans chaque cas qui se présente à notre observation ${ }^{59}$ ».

Le recours aux analyses de laboratoires tardera à se généraliser chez les praticiens privés. À partir de 1907, le test de BordetWassermann permet de dépister la syphilis mais la réponse des médecins est lente. Encore en 1918, le docteur Foucher se plaignait des résistances face à la réaction de Wassermann:

À ceux qui doutent encore de la valeur de cette réaction, je dirai que je la crois l'égale de la séro-réaction de Widal dans la fièvre typhoïde. Plus je m'en sers, plus je constate ce fait tous les jours. Elle est d'un usage courant dans tous les hôpitaux modernes et dans toutes les branches de la médecine. Bien entendu, pour qu'on puisse s'y fier, il faut qu'elle soit faite par un homme compétent, digne de foi et muni d'un bon outillage ${ }^{60}$.

Le médecin de pratique privée ne remplissait pas toujours ces trois conditions. Les analyses bactériologiques sont la plupart du temps prises en charge par le Conseil d'hygiène, les hôpitaux, les facultés médicales et les bureaux de santéé ${ }^{1}$. Avant que le bureau de santé de la ville de Québec ne se dote d'un laboratoire de bactériologie en 1911 sous la direction du docteur C.-O. Guimont, les analyses demandées par les médecins étaient confiées au laboratoire de l'Université Laval $^{62}$. La complexification des techniques et des équipements rendra

57. D. Goulet et O. Keel, «W. G. Johnston», op. cit.: «It was he who introduced the use of sterilized cotton wool swabs in test tubes for the taking of cultures», E.-H. Bensley, McGill Medical Luminaries (Montréal, McGill University, 1990), 60. 259.

58. A. Lesage, «L'odyssée d'une typhoïdique», L'Union médicale du Canada (1902):

59. Ibid., 263.

60. L'Union médicale du Canada (1918): 212. Le Royal Victoria Hospital introduit le test Bordet-Wassermann en 1907. Cf. Lewis, op. cit., 119.

61. Le Gouvernement fédéral publiera en 1920 trois brochures sur le diagnostic des maladies vénériennes: Les maladies vénériennes. Le test de Wassermann/Venereal Diseases. The Wassermann Test; Les maladies vénériennes. Les examens microscopiques/Venereal Diseases. The Microscopic Examination, et Les maladies vénériennes. Diagnostic et traitement/Venereal Diseases. Diagnosis and Treatment. Cf. J. Cassel, The Secret Plague. Venereal Disease in Canada 1838-1939 (Toronto, University of Toronto Press, 1987), 272.

62. R. Lemoine «La santé publique: de l'inertie municipale à l'offensive hygiéniste», $\mathrm{La}$ Ville de Québec, histoire municipale, tome 4: de la Confédération à la charte de 1929 (Québec, La Société historique de Québec, 1983), 180. 
nécessaire l'organisation de centres de diagnostic établis la plupart du temps au sein des institutions hospitalières et des organismes gouvernementaux d'hygiène publique ${ }^{63}$.

Certains médecins initiés aux procédés de laboratoire s'efforceront de convaincre leurs confrères de l'opportunité de se familiariser avec certaines analyses et de se doter d'un petit équipement de laboratoire. Lors du congrès de l'Association des médecins de langue française tenu en 1906, le docteur C.-A. Daigle, professeur de la succursale de Laval à Montréal, souligne que: «Le diagnostic est la pierre de touche du praticien. Or, la pratique de la médecine devenant de plus en plus une science et un art véritables, ayant des données précises [...] le médecin moderne doit, dans la mesure du possible, utiliser toutes les ressources que la science nouvelle met à sa disposition $^{64} . »$

Le docteur Daigle se fait le porte-parole d'un courant médical qui prendra de plus en plus d'importance durant les premières décennies de notre siècle et qui insiste sur la nécessité chez les médecins de pratique privée d'accroître les liens entre les sciences de laboratoire (chimie, physique, bactériologie) et la médecine clinique. Aussi, le docteur Daigle défend-il l'idée que: «tout praticien outillé doit posséder chez lui au moins un embryon de laboratoire» [et que] «dans tous les centres de quelque importance, il suffirait d'une entente entre les praticiens de l'endroit et du voisinage pour assurer l'organisation d'un laboratoire de recherches destiné à l'exécution des travaux longs ou compliqués qui ne peuvent se faire à domicile ${ }^{65}$.»

Parallèllement aux laboratoires des grands hôpitaux québécois, qui, la plupart du temps sont surchargés faute d'équipements adéquats et de personnel suffisant et ne peuvent répondre aux demandes extérieures, les laboratoires clu Conseil d'hygiène offraient une multitude

63. Le laxisme de certaines autorités municipales retardait l'utilisation des examens de laboratoire. À la suite du décès en 1922 de son bactériologiste, le docteur $\mathrm{H}$. Larouche, le laboratoire municipal de la ville de Montréal demeurera sans bactériologiste jusqu'en 1927. Les autorités médicales demeurent elless aussi lentes à réagir puisque la Société médicale de Montréal n'adresse que le 16 novembre 1926 une résolution enjoignant les autorités à embaucher sans délai un nouveau bactériologiste. Néanmoins le texte de la résolution adoptée par une société regroupant près de 300 médecins ne laisse guère d'équivoque quant à l'importance désormais accordée aux analyses bactériologiques. Voici l'une des résolutions adoptées: «Considérant que la nomination d'un bactériologiste est nécessaire et urgente, pour le contrôle des maladies contagieuses dans la Cité, ainsi que pour la protection du pauvre qui ne peut payer les frais d'une analyse bactériologique ou biologique indispensable au diagnostic de sa maladie quand il est traité en clientèle privée ou dans un dispensaire gratuit», Résolution de la Société médicale de Montréal, 11 novembre 1926.

64. C. A. Daigle, op. cit., 503-504.

65. Ibid., 504. 
d'analyses - sang, crachats, exsudats, fèces, urines, lait, eau, etc. aux médecins des villes et des campagnes. Le recours à ces analyses s'était certes déjà implanté durant les années 1920 mais c'est surtout durant la décennie 1930 que les praticiens y auront recours sur une large échelle. Les examens pratiqués au Conseil d'hygiène entre 1928 et 1930, s'accroissent de 57232 à 80770 ; en 1940, le nombre avait plus que triplé avec 285081 examens. Trois ans plus tard, le chiffre s'élèvera à 512305 . On le constate, le laboratoire d'analyse biomédicale du Conseil d'hygiène était devenu un appendice important de l'investigation clinique.

\section{Les premières analyses bactériologiques en milieu hospitalier}

À partir des années 1900 , les laboratoires biomédicaux constitueront aussi des équipements obligés des grands hôpitaux ${ }^{66}$. Certaines analyses avaient déjà cours dans plusieurs hôpitaux québécois durant les décennies précédentes mais elles se bornaient le plus souvent à un examen, à l'aide de réactifs, du taux d'albumine dans les urines, à quelques analyses sanguines ${ }^{67}$ sommaires et aux investigations anatomo-pathologiques. Le laboratoire médical en milieu hospitalier deviendra à plus ou moins long terme un outil essentiel pour assister les praticiens dans l'établissement du diagnostic, préciser les observations post-mortem du pathologiste et initier les étudiants aux nouveaux paramètres de la pratique clinique ${ }^{68}$. Or, les analyses bactériologiques contribueront grandement à favoriser l'essor des laboratoires.

Alors que généralement, le but avoué de la mise sur pied de petits laboratoires était de "procurer aux élèves des études cliniques

66. À partir de 1882, l'Hôtel-Dieu de Québec possède un petit laboratoire constitué d'un microscope, de flacons de réactif et d'une lampe à alcool. L'Hôtel-Dieu de Montréal possédait aussi au tournant des années 1880 un petit laboratoire. Un nouveau laboratoire plus spacieux et mieux équipé sera aménagé en 1886. En 1893, le Royal Victoria Hospital procède à l'installation d'un laboratoire clinique dirigé par le docteur Bruère où sont pratiquées quelques analyses biochimiques et bactériologiques. Quatre ans plus tard, la faculté de médecine de l'Université Laval procède à l'installation d'un laboratoire de chimie médicale. Cf. F. Rousseau, La Croix et le scalpel. Histoire des Augustines et de l'Hôtel-Dieu de Québec (Québec, Septentrion, 1989), 285; Sclater Lewis, ibid., 119 et 139; D. Goulet et A. Paradis, Trois siècles d'histoire médicale au Québec (1639-1939) (Montréal, VLB éditeur, 1992), 461 et 468.

67. Selon le témoignage de J.-E. Dubé, au début des années 1890 , «les analyses d'urine à l'Hôtel-Dieu étaient faites par le seul interne de service au-dessus du lavabo de sa chambre à coucher». "Nos Hôpitaux», L'Union médicale du Canada (1932), 155.

68. Le docteur Hervieux déclarait lors d'un discours prononcé en 1907 à l'École de médecine et de chirurgie/Faculté de médecine de Laval à Montréal que «la tendance actuelle est de diminuer les cours théoriques et d'augmenter les cours pratiques aux laboratoires et à l'hôpital». Discours rapporté par A. Lesage dans L'Union médicale du Canada (1947), 1302. 
pratiques en rapport avec les malades qu'ils voient journellement ${ }^{69}$ ", la pratique clinique et chirurgicale amorce au tournant du $\mathrm{XX}^{\mathrm{e}}$ siècle un tournant méthodologique qui affectera profondément l'organisation interne des hôpitaux. La nouvelle légitimité acquise par la médecine de laboratoire est clairement indiquée lors de l'allocution donnée par le surintendant de l'Hôpital Notre-Dame, le docteur E.-P. Lachapelle, en 1897-1898:

L'étudiant en médecine, après avoir suivi les leçons du maître au lit du malade, pourra, au laboratoire, interroger les sécrétions et les tissus pathologiques et constater de visu que les lésions signalées étaient bien exactes, cultiver des microbes et se convaincre par des inoculations aux animaux, que les germes incriminés produisaient bien les symptômes décrits et observés. Le médecin lui-même pourra se livrer à des recherches spéciales qui très souvent éclaireront son diagnostic, très souvent aussi dirigeront son traitement ${ }^{70}$.

De telles dispositions constituent en quelque sorte les conditions d'une rupture avec les pratiques antérieures. N'était-ce là que vœux pieux? En partie. La médecine hospitalière telle qu'elle se pratique dans la plupart des grands centres hospitaliers du Québec se situe à mi-chemin entre une médecine anatomo-clinique et une médecine de laboratoire. Les examens de laboratoire se font sur une base plus régulière dans le cas des malades privés mais demeure une pratique plutôt marginale vis-à-vis les patients des salles communes ${ }^{71}$. Les activités diagnostiques au sein des centres hospitaliers ne tarderont cependant pas à s'accentuer de façon importante durant les trois premières décennies de notre siècle. De grands hôpitaux généraux tels que le Montreal General Hospital, le Royal Victoria Hospital, l'Hôpital Notre-Dame ou les Hôtel-Dieu de Montréal et de Québec de même que des hôpitaux plus modestes, tels que l'Hôpital de la maternité, ont largement intégré les investigations bactériologiques à leur méthodologie clinique et à leurs activités de laboratoire. Or, dès le tournant du siècle, ces grands hôpitaux québécois avaient accueilli les «hommes-relais» de la bactériologie: Marien et Dubé sont engagés à l'Hôtel-Dieu de Montréal, Parizeau, Bernier, Derome, Lesage à l'Hôpital Notre-Dame, Rousseau à l'Hôtel-Dieu de Québec, Daigle à

69. Rapport annuel de l'Hôpital Notre-Dame (1887-1888), 15.

70. Ibid., 8 .

71. En 1898 un comité est formé pour établir un tarif des contributions pour les examens de laboratoire dans les cas des examens privés. Procès-Verbaux du bureau médical de l'Hôpital Notre-Dame, 7 décembre 1898. 
la Maternité et à la Crèche de la Miséricorde, Johnston au Montreal General Hospital, Adami et Bruère ${ }^{72}$ au Royal Victoria Hospital. L'hôpital, structure fondamentale de l'enseignement clinique, constituait un lieu privilégié pour promouvoir et transmettre les nouveaux savoirs bactériologiques. La présence de ces jeunes médecins réorientera certains aspects de la pratique hospitalière vers les nouveaux acquis de la bactériologie. Les membres des bureaux médicaux de ces institutions n'étaient pas tous enthousiastes mais tout de même la plupart reconnaissait la nécessité d'utiliser les moyens scientifiques disponibles pour l'observation des malades ${ }^{73}$.

Au tournant des années 1920, l'installation et l'instrumentation des laboratoires de bactériologie se sont améliorées mais elles demeuraient encore parfois largement inadéquates. Ainsi en est-il du laboratoire de bactériologie du docteur Bertrand à l'Hôpital NotreDame. Le docteur Bruère, nommé bactériologiste du Royal Victoria Hospital en 1915 travaille dans un petit laboratoire dont le financement est inadéquat ${ }^{74}$. Son premier assistant à temps plein, le docteur W. W. Beattie, ne sera engagé qu'en 1926. Le surintendant du Montreal General Hospital se plaint en 1923 de l'étroitesse des locaux alors que les laboratoires ne peuvent accueillir qu'un ou deux chercheurs à la fois ${ }^{75}$. De même, les laboratoires des Hôtels-Dieu de Québec et de Montréal sont loin d'offrir les locaux et les équipements nécessaires pour répondre à la demande croissante d'analyses bactériologiques et biochimiques. Quant aux laboratoires des petits hôpitaux de province, ils manquent de tout: personnel, équipements, locaux, etc. La situation est telle que le professeur Bourgeois de la faculté de médecine de l'Université de Montréal, déplorant leur «manque d'organisation scientifique», suggère que la faculté "relie ces laboratoires aux siens ${ }^{76}$ ». Tout de même, en 1937, le Québec se classera au premier rang au Canada pour la proportion des laboratoires cliniques dans les hôpitaux publics, soit $66,6 \%(56 / 84)^{77}$.

\section{CONCLUSION}

Notre étude remet en question l'idée défendue par R. Duchesne selon laquelle au Québec, à la fin du $\mathrm{XIX}^{\mathrm{e}}$ siècle,

72. Le docteur Bruère devient directeur des laboratoires du Royal Victoria Hospital en 1899. S. Lewis, op. cit., 119.

73. Rapport annuel de l'Hôpital Notre-Dame (1911), 28.

74. S. Lewis, op. cit., 220.

75. Ibid., 105-106.

76. Procès-verbaux de l'exécutif de la faculté de médecine de l'Université de Montréal, 23 novembre 1932, 187.

77. Annuaire du Canada 1939 (Ottawa, J.-O. Patenaude, 1940), 1084. 
les (médecins) francophones sont un petit peu en retard par rapport à (la) modernisation de la médecine [...] Les idées modernes au sujet de la bactériologie, au sujet de l'asepsie et de l'antisepsie en chirurgie tardent un petit peu à s'imposer. Il y a des raisons assez évidentes à cela, c'est que les contacts sont moins fréquents, moins étroits avec les médecins européens et américains, qu'ils ne le sont pour leurs collègues anglophones ${ }^{78}$.

Nous avons montré le rôle joué par de jeunes médecins francophones initiés à la pratique bactériologique durant les décennies 1890,1900 et 1910 dans la mise en place des pratiques médicales bactériologiques, lesquelles ne tarderont pas à orner le paysage médical québécois. On ne peut considérer qu'il y ait eu retard de la médecine francophone québécoise en ce qui regarde l'introduction et l'implantation de la bactériologie. Bien au contraire, les médecins québécois inscrits à l'Institut Pasteur ou dans les centres hospitaliers parisiens ont constitué une avant-garde importante qui a largement contribué - par la diffusion des pratiques bactériologiques, par l'enseignement, la pratique hospitalière, la publication d'articles et la présentation de communications - à enseigner et à vulgariser les théories pasteuriennes et à modifier certaines pratiques hygiéniques, prophylactiques et thérapeutiques au profit des pratiques bactériologiques. Ils ont par ailleurs le mérite d'avoir favorisé l'implantation d'une médecine de laboratoire en territoire québécois.

Il ne faut pas non plus, à notre avis, mesurer le «retard» de l'élite de la médecine francophone, comme le fait Duchesne, à l'aune de l'idéologie «oslérienne» qui prône, à la fin du XIX ${ }^{\mathrm{e}}$ siècle, une médecine axée sur la clinique, le laboratoire et la recherche scientifique. Ces prescriptions - du reste, loin d'être rigoureusement suivies par l'ensemble de la profession médicale anglophone du Québec (Hingston, Fenwick, Shepherd, Nelson, Armstrong, Ahern, etc.) - sont en grande partie défendues, du moins en ce qui regarde la clinique et le laboratoire, par plusieurs jeunes médecins francophones (Bourgeois, Saint-Jacques ${ }^{79}$, Daigle, Harwood, Mayrand, Hervieux, Bédard, Vallée, Aubry, Bernier, Parizeau, Mercier, Lesage, Marien, etc.) ainsi que par certains de leurs aînés (E.-P. Lachapelle, Foucher, Desrosiers, Guérin, Benoit) au moment où, de l'aveu même

78. R. Duchesne, «L'émergence de la recherche biomédicale moderne», Petite histoire de la médecine au Québec (Montréal, Société Radio-Canada (Les transcriptions Radio), 1989), 9.1.

79. Le docteur E. Saint-Jacques est certes un médecin sensibilisé aux nouvelles prescriptions de la médecine scientifique puisqu'après avoir effectué un séjour d'un an dans un hôpital de New York vers 1897, il se rend pour un séjour de plus de deux ans en France et en Allemagne où il suit les cliniques et les cours de Tillaux, Tuffier, Pozzi, Letulle et Segond à Paris ainsi que ceux de Virchow, Bergmann et Koerte à Berlin et de Trendelenberger à Leipzig. 
de Duchesne, cet idéal «prend racine au Canada, à peu près au tournant du siècle ${ }^{80} \gg$. Or, précisément à ce moment-là, nous avons montré que se mettent en place dans les grands hôpitaux francophones Hôtels-Dieu de Québec et de Montréal, Hôpital Notre-Dame - de petits laboratoires cliniques. Autre reflet de l'intérêt suscité par les travaux des pasteuriens, quelques médecins s'efforcèrent en 1906 de fonder un Institut Pasteur au Québec. L'Association des médecins de langue française de l'Amérique du Nord avait en effet décidé, lors de son congrès tenu à Trois-Rivières, de former un comité composé des docteurs Loir de l'Institut Pasteur de Paris, Brochu de Québec, Foucher de Montréal et Panneton de Trois-Rivières pour étudier la possibilité de fonder un tel institut au Québec. Était alors envisagé d'ajouter aux activités d'analyse et de production de vaccins des activités de recherche. Mais cette initiative n'aura pas de suite. Avec l'ampleur des problèmes liés à la tuberculose, aux maladies vénériennes, aux maladies épidémiques, à la mortalité infantile et les multiples tâches médicales - enseignement, pratique privée, pratique hospitalière, pratique philanthropique - cumulées par la majorité des pionniers de la médecine bactériologique, bien peu de médecins avaient le temps nécessaire pour se vouer à la mise sur pied d'un institut qui aurait demandé temps et argent. De plus, on considérait que le Conseil provincial d'hygiène remplissait bien les tâches les plus urgentes d'approvisionnement et d'analyses.

Il est cependant vrai, comme le souligne pertinemment Duchesne, que la recherche biomédicale demeurera à peu près inexistante en milieu francophone jusqu'à la fin des années 1930. Le plus souvent, les pratiques de laboratoire ne seront jusque-là considérées que comme un appendice de l'investigation clinique. Il faut tout de même souligner l'exception notable que constitue les précoces travaux de recherche clinique sur le BCG entrepris dès 1925 à l'Université de Montréal par le docteur Beaudoin en collaboration avec le docteur Pettit, délégué de l'Institut Pasteur à Paris ${ }^{81}$. Ces travaux sont entrepris au moment où, selon le témoignage du docteur Frappier, le vaccin BCG rencontrait les plus dures oppositions «dans plusieurs laboratoires de microbiologie parmi les plus renommés d'Amérique ${ }^{82} »$. Mais peu familier avec la méthode expérimentale, les travaux de Baudouin demeurèrent circonscrits aux aspects épidémiologiques et statistiques.

80. R. Duchesne, op. cit., 9.1.

81. Voir A. Frappier, op. cit., «Cinquante ans d'étude et d'emploi du vaccin BCG au Canada», 75-96.

82. A. Frappier, op. cit., 65. 
Du reste, les leçons de bactériologie, jusqu'aux années 1930, étaient le plus souvent considérées comme des cours subalternes d'enseignement pratique en relation étroite avec les cours d'hygiène publique ou liées aux traditionnelles et prestigieuses chaires d'histologie, de pathologie et de thérapeutique médicale. La bactériologie comme matière autonome d'enseignement universitaire demeurera mal logée au sein des facultés médicales. La plupart des titulaires de ces chaires envisageait la bactériologie davantage comme une technique que comme un champ de recherche. Du moins est-ce le plus souvent le cas des professeurs des facultés médicales de Laval et de Montréal, engagés à temps partiel, qui associent leur enseignement universitaire et clinique à leur pratique privée. En outre, les laboratoires demeuraient mal outillés et les fonds de recherche peu accessibles. En conséquence, les travaux bactériologiques demeureront essentiellement associés aux activités d'identification des microorganismes et aux activités restreintes et ponctuelles de production de vaccins ou de sérums. Les pratiques médicales bactériologiques avaient incontestablement contribué à légitimer et à populariser ce nouveau champ de savoir mais celui-ci demeurait en position subalterne et se voyait le plus souvent confiné à des activités prophylactiques, diagnostiques et thérapeutiques étroitement encadrées par la profession médicale. La bactériologie, alors en voie de disciplinarisation, devait se détacher des facultés médicales pour atteindre sa pleine autonomie et accentuer ses fonctions de recherche et de production. Le docteur Frappier l'avait compris ${ }^{83}$. Dès 1936, il avait demandé la création d'un certificat spécialisé de bactériologie à l'Université de Montréal, prélude à la fondation de l'Institut de microbiologie et d'hygiène. On connaît le rôle que cette institution jouera par la suite dans les processus d'institutionnalisation de la bactériologie et dans le développement scientifique francophone au Québec.

83. Voir à ce propos, A. Frappier, op. cit. et L. Chartrand et al., op. cit., «Armand Frappier et l'Institut de microbiologie de Montréal», 362-365. 